\title{
TORSION IN THE COHOMOLOGY OF LINE BUNDLES ON HOMOGENEOUS SPACES FOR CHEVALLEY GROUPS
}

\author{
HENNING HAAHR ANDERSEN
}

\begin{abstract}
In this paper we study the torsion submodules of the cohomology modules for line bundles on $G / B$ where $G$ is a Chevalley group over $\mathbf{Z}$ and $B$ a Borel subgroup.
\end{abstract}

Let $G$ be a Chevalley group over $\mathbf{Z}$ and let $B$ be a Borel subgroup of $G$. By $H^{i}(\lambda)$ (resp. $H_{t}^{i}(\lambda), H_{f}^{i}(\lambda)$ ) we denote the $i$ th cohomology group (resp. its torsion submodule, free quotient) of the line bundle on $G / B$ induced by the character $\lambda$ of $B$. In [3] we studied the modules $H_{f}^{i}(\lambda)$ and used them to construct "Jantzen-type" filtrations of $H_{f}^{i}(\lambda) \otimes k$ where $k$ is a field of characteristic $p>0$.

In this paper we shall study the torsion modules $H_{t}^{i}(\lambda)$. We shall prove for instance that $H_{t}^{1}(\lambda)=0$ for all $\lambda$. (The vanishing of $H_{t}^{0}(\lambda)$ and $H_{t}^{N}(\lambda), N$ being the dimension of $G / B$, is a consequence of Kempf's vanishing theorem, see Corollary 2.6(ii) of [3].) More generally, we find an integer $n=n(i, \lambda)$ such that the $p$-torsion in $H^{i}(\lambda)$ is killed by $p^{n}$. It should be pointed out though that (due to the fact that no generalization of Bott's theorem to positive characteristic is known) the vanishing behavior of $H_{t}^{i}(\lambda)$ remains very much a mystery in general.

Although we tried to ignore the torsion submodule of $H^{\circ}(\lambda)$ in [3] our sum formula there (Theorem 4.10) contains two terms which come from $H_{t}^{*}(\lambda)$. Of course in the generic case (in particular for $\lambda$ either dominant or antidominant) these terms vanish. In the general case we shall show in this paper that the $H_{t}^{i}(\lambda)$ 's also have natural filtrations and that the sum formula for the corresponding filtrations of $H_{t}^{i}(\lambda) \otimes k$ accounts for these extra terms in Theorem 4.10 of [3].

We illustrate our results in the last section by working out explicitly the semisimple rank 2 case. For these groups we have given in $[2, \S 5]$ a description of the vanishing behaviour for $H_{k}^{*}(\lambda)$.

\section{Torsion in $H^{*}(\lambda)$.}

1.1. In addition to the notation already introduced above we shall use the following: By $T$ we denote a (split) maximal torus contained in $B$ and we let $X(T)$ be its character group. The root system associated to $(G, T)$ is denoted $R$ and we choose a set of simple roots $S$ such that $B$ corresponds to the negative roots. Recall that the dot action of the Weyl group $W$ is given by $w \cdot \lambda=w(\lambda+\rho)-\rho$ for $w \in W, \lambda \in X(T)$. Here $\rho$ is half the sum of the positive roots.

Received by the editors November 7, 1984 and, in revised form, April 5, 1985.

1980 Mathematics Subject Classification. Primary 20G10; Secondary 14M17. 
If $A$ is a commutative ring then we let $G_{A}$ (resp. $B_{A}$ ) denote the algebraic group over $A$ corresponding to $G$ (resp. $B$ ) and we let $H_{A}^{-}(\lambda)$ denote the cohomology of the line bundle on $G_{A} / B_{A}$ induced by the character $\lambda$ of $B$ (or $B_{A}$ ). Here and in the following, it is understood that $\lambda$ denotes the action of $B$ (resp. $B_{A}$ ) on $\mathbf{Z}$ (resp. $A$ ) given by $\lambda$. From [3, Theorem 1.18] we recall the universal coefficient

THEOREM. For each $i$ there is an exact sequence of $G_{A}$-modules

$$
0 \rightarrow H^{i}(\lambda) \otimes A \rightarrow H_{A}^{i}(\lambda) \rightarrow \operatorname{Tor}_{1}^{\mathbf{Z}}\left(H^{i+1}(\lambda), A\right) \rightarrow 0 .
$$

1.2. Proposition. For any character $\lambda$ of $B$ the cohomology group $H^{1}(\lambda)$ is torsion free.

Proof. Suppose first $\lambda$ is dominant (i.e. $\left\langle\alpha^{v}, \lambda\right\rangle \geqslant 0$ for all $\alpha \in S$ ). Then by Kempf's theorem $H_{K}^{1}(\lambda)=0$ for any field $K$ and hence by Theorem $1.1 H^{1}(\lambda)=0$. On the other hand if $\lambda$ is not dominant then it is well known that $H_{K}^{0}(\lambda)=0$ for any field $K$. So in this case Theorem 1.1 shows that $\operatorname{Tor}_{1}^{\mathbf{Z}}\left(H^{1}(\lambda), K\right)=0$ for any field $K$, i.e., $H^{1}(\lambda)$ is torsion free.

1.3. By Proposition 1.2 and Theorem 1.1 we see that $H^{1}(\lambda) \neq 0$ if and only if $H_{\mathbf{Q}}^{1}(\lambda) \neq 0$. Hence by Bott's theorem we obtain

Corollary. Let $\lambda \in X(T)$. Then $H^{1}(\lambda) \neq 0$ if and only if there exists $\alpha \in S$ such that $s_{\alpha} \cdot \lambda$ is dominant.

1.4. From now on let $p$ be a fixed prime and let $\nu_{p}: \mathbf{N} \rightarrow \mathbf{N}$ denote $p$-adic valuation, i.e., $\nu_{p}(n)=m$ if $n=p^{m} d$ with $d$ prime to $p$.

We also fix $\lambda \in X(T)$ such that $\lambda+\rho$ is dominant. For each positive root $\alpha$ with $\left\langle\alpha^{v}, \lambda+\rho\right\rangle>0$ we let $r_{\alpha} \in \mathbf{Z}$ be determined by the inequalities $p^{r_{\alpha}} \leqslant\left\langle\alpha^{v}, \lambda+\rho\right\rangle$ $\left\langle p^{r_{\alpha}+1}\right.$ and we set $n_{\alpha}=r_{\alpha}-v_{p}\left(\left\langle\alpha^{v}, \lambda+\rho\right\rangle\right)$. If $\left\langle\alpha^{v}, \lambda+\rho\right\rangle=0$ we set $n_{\alpha}=0$.

As usual when $w \in W$ we denote its length by $l(w)$.

Proposition. Let $w \in W$ and suppose $w=s_{\beta_{1}} \cdots s_{\beta_{i}}$ is a reduced expression for w. Set $\alpha_{m}=s_{\beta_{i}} \cdots s_{\beta_{m+1}}\left(\beta_{m}\right)$ for $m=i, i-1, \ldots, 1$. Then

(i) For $j \leqslant l(w)$ the p-torsion in $H^{j}(w \cdot \lambda)$ is killed by $p^{n}$ for $n \geqslant n(j)=\sum_{m=1}^{j-1} n_{\alpha_{m}}$.

(ii) For $j>l(w)$ the p-torsion in $H^{j}(w \cdot \lambda)$ is killed by $p^{n}$ for $n \geqslant n(w)=n(i)=$ $\sum n_{\alpha}$, the sum being over $\left\{\alpha \in R_{+} \mid w(\alpha) \in R_{-}\right\}$.

Proof. Let $j \leqslant l(w)$ and set $w_{j}=s_{\beta_{j-1}} \cdots s_{\beta_{1}}$. Recall from [3, §4] that we have homomorphisms

$$
H^{j}(w \cdot \lambda) \stackrel{T_{w_{j}}}{\rightarrow} H^{1}\left(w_{j} w \cdot \lambda\right) \stackrel{T_{w-1}}{\rightarrow} H^{j}(w \cdot \lambda),
$$

whose composite is $d p^{n(j)}$ for some $d$ prime to $p$. (In [3] we only considered these homomorphisms over the localization of $\mathbf{Z}$ at $p$ but it is clear from their construction that they actually exist over $\mathbf{Z}$.) Now we have just proved (Proposition 1.2) that $H^{1}\left(w_{j} w \cdot \lambda\right)$ is torsion free. Hence (i) follows. For $j>l(w)$ we consider the 
composite

$$
H^{j}(w \cdot \lambda) \stackrel{T_{w^{-1}}}{\rightarrow} H^{j-l(w)}(\lambda) \stackrel{T_{w^{*}}}{\rightarrow} H^{j}(w \cdot \lambda)
$$

and use that by Kempf's theorem $H^{n}(\lambda)=0$ for $n>0$.

Remark. Note that if $\left\langle\alpha_{j}^{v}, \lambda+\rho\right\rangle=0$ for some $j$ then by our definitions $n_{\alpha}=0$. However, in this case $H^{\cdot}\left(w_{j} w \cdot \lambda\right)=0$ and as pointed out below this allows us to improve the proposition a bit.

1.5. Suppose $H_{t}^{i^{\prime}}\left(w^{\prime} \cdot \lambda\right)=0$ for some $i^{\prime}, w^{\prime}$. By considering the maps

$$
H^{i^{\prime}+l\left(w w^{\prime}\right)}(w \cdot \lambda) \stackrel{T_{w^{\prime} w^{-1}}}{\rightarrow} H^{i^{\prime}}\left(w^{\prime} \cdot \lambda\right) \stackrel{T_{w w^{\prime}-1}}{\rightarrow} H^{i^{\prime}+l\left(w^{\prime} w^{-1}\right)}(w \cdot \lambda),
$$

we see that the $p$-torsion in $H^{i^{\prime}+l\left(w w^{\prime}\right)}(w \cdot \lambda)$ is killed by $p^{n\left(w^{\prime} w^{-1}\right)}$.

This observation can be used to improve the bound $n(j)$ from Proposition 1.4, e.g. if there exists $\alpha$ such that $\left\langle\alpha^{v}, \lambda+\rho\right\rangle=0$ (compare the remark at the end of 1.4) or if $j$ is close to $N$. In the latter case one uses the fact that $H_{t}^{i}\left(w^{\prime} \cdot \lambda\right)=0$ for $i \geqslant N$ and all $w^{\prime}$.

In the case where $n_{\alpha} \leqslant 1$ for all $\alpha$ we get in particular

The $p$-torsion in $H^{j}(w \cdot \lambda)$ is killed by $p^{n}$ for

$$
n \geqslant \min \{j-1, N-j\} \text {. }
$$

2. The sum formula. Let $k$ denote a field of characteristic $p$ (fixed for the rest of this paper).

In this section we start by expressing the character of the $T_{k}$-modules $H_{t}^{i}(\lambda) \otimes k$ in terms of the character of $H_{k}^{*}(\lambda)$. Then we shall consider a filtration of $H_{t}^{i}(\lambda) \otimes k$ and derive a sum formula for it.

In this section $\lambda$ will denote a character of $T$ such that $\lambda+\rho$ is dominant.

2.1. For any finitely generated $T$ or $T_{k}$-module $V$ we let $\operatorname{ch} V$ denote the formal character of $V$.

Proposition. Let $w \in W$ and $n \in \mathbf{N}$. Then

$$
\operatorname{ch}\left(H_{t}^{i}(w \cdot \lambda) \otimes k\right)= \begin{cases}\sum_{j \geqslant i}(-1)^{j-i} \operatorname{ch} H_{k}^{j}(w \cdot \lambda) & \text { if } i>l(w), \\ \sum_{j<i}(-1)^{i-j-1} \operatorname{ch} H_{k}^{j}(w \cdot \lambda) & \text { if } i \leqslant l(w) .\end{cases}
$$

Proof. Note first that according to [3, Lemma 2.8] we have

$$
\operatorname{ch}\left(H_{i}^{j}(w \cdot \lambda) \otimes k\right)=\operatorname{ch} \operatorname{Tor}_{1}^{\mathbf{Z}}\left(H^{j}(w \cdot \lambda), k\right) \text { for all } j .
$$

Using this together with the fact that $H^{j}(w \cdot \lambda)=H_{t}^{j}(w \cdot \lambda)$ for $j \neq l(w)[3$, Corollary 2.6(i)] we deduce from Theorem 1.1 that

$$
\operatorname{ch}\left(H_{t}^{j}(w \cdot \lambda) \otimes k\right)=\operatorname{ch} H_{k}^{j}(w \cdot \lambda)-\operatorname{ch}\left(H_{t}^{j+1}(w \cdot \lambda) \otimes k\right) .
$$

The proposition now follows by induction on $i$ (descending for $i>l(w)$, ascending for $i \leqslant l(w))$. 
2.2. When $A$ is a finite abelian $p$-group we set

$$
n(A)=\min \left\{n \mid p^{n} A=0\right\} \text {. }
$$

We then have a filtration of $A$

$$
A=A^{0} \supset A^{1} \supset \cdots \supset A^{n(A)}=0
$$

of length $n(A)$ given by $A^{i}=p^{i} A$. Let us write $n=n(A)$. Multiplication by $p^{i}$ is a surjection from $A$ onto $A^{i}$ and we let $A_{k}^{n-i}$ denote the kernel of the induced map $A \otimes k \rightarrow A^{i} \otimes k$, i.e., we have the short exact sequence

$$
0 \rightarrow A_{k}^{n-i} \rightarrow A \otimes k \rightarrow A^{i} \otimes k \rightarrow 0 .
$$

Then $A \otimes k=A_{k}^{0} \supset A_{k}^{1} \supset \cdots \supset A_{k}^{n(A)}$ is a filtration of $A \otimes k$ and we have

$$
\operatorname{dim} A_{k}^{n-i}=\operatorname{dim}(A \otimes k)-\operatorname{dim}\left(A^{i} \otimes k\right) .
$$

Noting that $A^{i} \otimes k \cong A^{i} / A^{i+1} \otimes_{\mathrm{z} / p} k$ we have

$$
\operatorname{dim}\left(A^{i} \otimes k\right)=\nu_{p}\left(\operatorname{ord}\left(A^{i}\right)\right)-\nu_{p}\left(\operatorname{ord}\left(A^{i+1}\right)\right) .
$$

Combining (1) and (2) we obtain

$$
\sum_{i \geqslant 1} \operatorname{dim} A_{k}^{i}=n(A) \operatorname{dim}(A \otimes k)-\nu_{p}(\operatorname{ord}(A)) .
$$

Clearly, if $A$ is a $G$ (resp. $T$ )-module then the above filtration of $A \otimes k$ is a filtration by $G_{k}$ (resp. $T_{k}$ )-submodules. Applying (3) to the individual weight spaces we get then

$$
\sum_{i \geqslant 1} \operatorname{ch} A_{k}^{i}=n(A) \operatorname{ch}(A \otimes k)-\sum_{\mu} \nu_{p}\left(\operatorname{ord} A_{\mu}\right) e^{\mu}
$$

By applying the above to the case where $A$ is the $p$-torsion part of $H^{i}(w \cdot \lambda)$ we have

Proposition. Let $w \in W, i \in \mathbf{N}$ and set

$$
n(i, w)=\min \left\{n \mid p^{n} \text { kills the p-torsion in } H^{i}(w \cdot \lambda)\right\} .
$$

Then we have a filtration

$$
H_{t}^{i}(w \cdot \lambda) \otimes k=H_{t}^{i, 0}(w \cdot \lambda) \supset H_{t}^{i, 1}(w \cdot \lambda) \supset \cdots
$$

of length $n(i, w)$ by $G_{k}$-modules satisfying

$$
\sum_{j \geqslant 1} \operatorname{ch} H_{i}^{i, j}(w \cdot \lambda)=n(i, w) \operatorname{ch}\left(H_{t}^{i}(w \cdot \lambda) \otimes k\right)-\sum_{\mu} \nu_{p}\left(\operatorname{ord} H_{t}^{i}(w \cdot \lambda)_{\mu}\right) e^{\mu} .
$$

2.3. In [3] we defined for each $w \in W$ an element $\theta_{w} \in \mathbf{Z}[X(T)]$ by the formula

$$
\theta_{w}=\sum_{i}(-1)^{i} \sum_{\mu} \nu_{p}\left(\operatorname{ord} H_{t}^{i}(w \cdot \lambda)_{\mu}\right) e^{\mu} .
$$

Now by Proposition 2.2 we can rewrite this as follows:

(1)

$$
\theta_{w}=\sum_{i}(-1)^{i} n(i, w) \operatorname{ch}\left(H_{t}^{i}(w \cdot \lambda) \otimes k\right)-\sum_{i}(-1)^{i} \sum_{j} \operatorname{ch} H_{i}^{i, j}(w \cdot \lambda) .
$$


As in [3] we set $\chi(\mu)=\sum_{i}(-1)^{i} \operatorname{ch} H^{i}(\mu)$ for $\mu \in X(T)$ and if $\alpha \in R$ we set $\operatorname{sgn}(\alpha)=1$ if $\alpha$ is a positive root, $\operatorname{sgn}(\alpha)=-1$ if $\alpha$ is a negative root. Let us define the element $\Sigma_{w}(\lambda) \in \mathbf{Z}[X(T)]$ by

(2) $\Sigma_{w}(\lambda)=\left(\sum_{\alpha \in R_{+} \cap w^{-1}\left(R_{+}\right)} n_{\alpha}\right) \chi(\lambda)+\sum_{\alpha \in R_{+}} \operatorname{sgn} w(\alpha) \sum \nu_{p}(m p) \chi(\lambda-m p \alpha)$,

where the last sum runs over $\left\{m \mid 0<m p<\left\langle\alpha^{v}, \lambda+\rho\right\rangle\right\}$. Note that $\chi(\mu)$ is given by Weyl's character formula so that $\Sigma_{w}(\lambda)$ involves only computable terms. Let as usual $w_{0}$ be the longest element in $W$.

Using (1) and (2) we can now reformulate the sum formula from [3, Theorem 4.10] (where the equality sign is missing!).

THEOREM. The filtration $\left(H_{k}^{\prime(w)}(w \cdot \lambda)^{j}\right)_{j \geqslant 0}$ from $[3, \S 4]$ and the filtrations $\left(H_{t}^{i, j}(w \cdot \lambda)\right)_{j \geqslant 0}$ from 2.3 above satisfy the following sum formula:

$$
\begin{aligned}
& \sum_{j>0} \operatorname{ch} H_{k}^{l(w)}(w \cdot \lambda)^{j} \\
& +\sum_{i}(-1)^{i+l(w)}\left(\sum_{j \geqslant 1} \operatorname{ch} H_{t}^{i, j}(w \cdot \lambda)-(-1)^{N} \operatorname{ch} H_{t}^{i, j}\left(w_{0} w \cdot \lambda\right)\right) \\
& \quad-(-1)^{l(w)} \sum_{i}(-1)^{i}\left(n(i, w) \operatorname{ch}\left(H_{t}^{i}(w \cdot \lambda) \otimes k\right) .\right. \\
& \left.\quad-(-1)^{N} n\left(i, w_{0} w\right) \operatorname{ch}\left(H_{t}^{i}\left(w_{0} w \cdot \lambda\right) \otimes k\right)\right)=\Sigma_{w}(\lambda) .
\end{aligned}
$$

2.4. Let still $w \in W$. From Proposition 2.1 we derive

$$
\sum_{i}(-1)^{i} \operatorname{ch}\left(H_{t}^{i}(w \cdot \lambda) \otimes k\right)=\sum_{j}(j-l(w))(-1)^{j} \operatorname{ch} H_{k}^{j}(w \cdot \lambda) .
$$

If we as in $[3,4.14]$ let $\phi$ denote the automorphism of $G$ corresponding to the root system automorphism $\alpha \mapsto-w_{0}(\alpha)$, then we have $\operatorname{ch}\left(V^{*}\right)=\operatorname{ch} V^{\phi}$ for any $G$-module $V$. In particular by Serre duality we get

$$
\operatorname{ch} H_{k}^{i}(w \cdot \lambda)=\operatorname{ch} H_{k}^{N-i}\left(w_{0} w \cdot \lambda\right) .
$$

Hence combining (1) and (2) we find

$$
\begin{aligned}
& \sum_{i}(-1)^{i}\left(\operatorname{ch}\left(H_{t}^{i}(w \cdot \lambda) \otimes k\right)-(-1)^{N} \operatorname{ch}\left(H_{t}^{i}\left(w_{0} w \cdot \lambda\right) \otimes k\right)\right) \\
& =2 \sum_{j}(j-l(w))(-1)^{k} \operatorname{ch} H_{k}^{j}(w \cdot \lambda) .
\end{aligned}
$$

Now if $n(i, w)=n\left(i, w_{0} w\right) \leqslant 1$ for all $i$ we get by inserting (3) in the formula in Theorem 2.3 (Note that $n(i, w)=0$ means that $H_{t}^{i}(w \cdot \lambda)=0$, i.e.,

$$
n(i, w) \operatorname{ch}\left(H_{t}^{i}(w \cdot \lambda) \otimes k\right)=\operatorname{ch}\left(H_{t}^{i}(w \cdot \lambda) \otimes k\right)
$$

also in that case.): 
COROllary. Suppose the p-torsion in $H^{\cdot t}(w \cdot \lambda)$ and in $H^{\cdot t}\left(w_{0} w \cdot \lambda\right)$ is killed by p. Then the sum formula reduces to

$$
\sum_{j \geqslant 1} \operatorname{ch} H_{k}^{l(w)}(w \cdot \lambda)^{j}-2(-1)^{l(w)} \sum_{j}(j-l(w))(-1)^{j} \operatorname{ch} H_{k}^{j}(w \cdot \lambda)=\Sigma_{w}(\lambda) .
$$

3. Rank 2. In this section we consider the case where $G$ has semisimple rank 2. In this case the vanishing behaviour of $H_{k}^{i}(w \cdot \lambda)$ is known, see [2, §5] (again $\lambda$ denotes a character with $\lambda+\rho$ dominant).

3.1. TYPE $A_{2}$. Our results in $\S 1$ show that in this case we have

$$
H_{t}^{i}(\mu)=0 \text { for all } \mu \in X(T), \text { for } i \neq 2 .
$$

Moreover, by 2.4 (1) we get

$$
\operatorname{ch}\left(H_{t}^{2}(w \cdot \lambda) \otimes k\right)= \begin{cases}\operatorname{ch} H_{k}^{2}(w \cdot \lambda) & \text { if } l(w)=1 \\ \operatorname{ch} H_{k}^{1}(w \cdot \lambda) & \text { if } l(w)=2 \\ 0 & \text { otherwise. }\end{cases}
$$

In particular, it follows that

There is nonzero $p$-torsion in $H^{2}(\nu)$ if and only if both $H_{k}^{1}(\nu)$ and $H_{k}^{2}(\nu)$ are nonzero.

Hence the $\nu \in X(T)$ for which there is nonzero $p$-torsion in $H^{2}(\nu)$ are those which belong to the shaded alcoves in Figure 1, p. 94 of [1].

Suppose now that $\left\langle\alpha^{v}, \lambda+\rho\right\rangle<p^{2}$ for all $\alpha \in R$. Then by Proposition 1.4 and 1.5 we see that the $p$-torsion in $H^{\circ}(w \cdot \lambda)$ is killed by $p$ for all $w$. Hence by Corollary 2.4 we get

$$
\sum_{j=1}^{3} \operatorname{ch} H_{k}^{1}(w \cdot \lambda)^{j}+2 \operatorname{ch} H_{k}^{2}(w \cdot \lambda)=\Sigma_{w}(\lambda) \quad \text { if } l(w)=1,
$$

and

$$
\sum_{j=1}^{3} \operatorname{ch} H_{k}^{2}(w \cdot \lambda)^{j}-2 \operatorname{ch} H_{k}^{1}(w \cdot \lambda)=\Sigma_{w}(\lambda) \quad \text { if } l(w)=2 .
$$

It may be added that in the cases where we in (4) (resp. (5)) have a nonzero contribution from $H_{k}^{2}(w \cdot \lambda)$ (resp. $H_{k}^{1}(w \cdot \lambda)$ ), it is known that this module is irreducible, see [1, Proposition 4.7]. Moreover, it is very easy to determine the submodule structure of both $H_{k}^{1}(w \cdot \lambda)$ and $H_{f}^{1}(w \cdot \lambda) \otimes k$ (resp. $H_{k}^{2}(w \cdot \lambda)$ and $\left.H_{f}^{2}(w \cdot \lambda) \otimes k\right)$ in these degenerate cases (the modules involve at most 4 composition factors). In the generic case $\mathrm{K}$. Kühne-Hausmann has worked out the submodule structure of $H_{k}^{l(w)}(w \cdot \lambda)$, [4, Chapter VI, Lemma 2], and her results together with the above show that for this group our filtration of $H_{f}^{l(w)}(w \cdot \lambda) \otimes k$ coincides always with the socle series provided $\lambda$ satisfies $\left\langle\alpha^{v}, \lambda\right\rangle\left\langle p^{2}-p\right.$ for all positive roots $\alpha$. (It is easy to check that this condition on $\lambda$ is necessary even for the statement to be true for Weyl modules.) 
3.2. TYPE $B_{2}$. Using the same methods as in 3.1 we obtain

$$
\begin{gathered}
H_{t}^{i}(\nu)=0 \quad \text { for all } \nu, \quad \text { for } i \neq 2,3 \\
\operatorname{ch}\left(H_{t}^{2}(w \cdot \lambda) \otimes k\right)= \begin{cases}\operatorname{ch} H_{k}^{2}(w \cdot \lambda) & \text { for } l(w)=1, \\
\operatorname{ch} H_{k}^{1}(w \cdot \lambda) & \text { for } l(w)=2, \\
0 & \text { otherwise. }\end{cases} \\
\operatorname{ch}\left(H_{t}^{3}(w \cdot \lambda) \otimes k\right)= \begin{cases}\operatorname{ch} H_{k}^{3}(w \cdot \lambda) & \text { for } l(w)=2, \\
\operatorname{ch} H_{k}^{2}(w \cdot \lambda) & \text { for } l(w)=3, \\
0 & \text { otherwise. }\end{cases}
\end{gathered}
$$

There is nonzero $p$-torsion in $H^{2}(\nu)$ (resp. $\left.H^{3}(\nu)\right)$ if and only if both $H_{k}^{1}(\nu)$ (resp. $\left.H_{k}^{3}(\nu)\right)$ and $H_{k}^{2}(\nu)$ are nonzero.

Hence the regions for which $H^{2}(\nu)$ (resp. $H^{3}(\nu)$ ) contains nonzero $p$-torsion are easily read off from Figure 1, p. 255 of [2].

Suppose now that $\left\langle\alpha^{v}, \lambda+\rho\right\rangle<p^{2}$ for all $\alpha \in R$. Then by Proposition 1.4 and 1.5 we see that the $p$-torsion in $H^{\circ}(w \cdot \lambda)$ is killed by $p$ for all $w$. Hence by Corollary 2.4 we get

$$
\sum_{j \geqslant 0} \operatorname{ch} H_{k}^{1}(w \cdot \lambda)^{j}+2 \operatorname{ch} H_{k}^{2}(w \cdot \lambda)=\Sigma_{w}(\lambda) \quad \text { if } l(w)=1,
$$

$$
\sum_{j \geqslant 1} \operatorname{ch} H_{k}^{2}(w \cdot \lambda)^{j}-2 \operatorname{ch} H_{k}^{1}(w \cdot \lambda)+2 \operatorname{ch} H_{k}^{3}(w \cdot \lambda)=\Sigma_{w}(\lambda) \quad \text { if } l(w)=2
$$

(note that if $H_{k}^{1}(w \cdot \lambda) \neq 0$ then $H_{k}^{3}(w \cdot \lambda)=0$ and vice versa),

$$
\sum_{j \geqslant 1} \operatorname{ch} H_{k}^{3}(w \cdot \lambda)^{j}-2 \operatorname{ch} H_{k}^{2}(w \cdot \lambda)=\Sigma_{w}(\lambda) \quad \text { if } l(w)=3 .
$$

In this case the nongeneric terms in these formulas are not always irreducible characters. In fact, the corresponding modules are not always semisimple, so that our filtrations of $H_{t}^{*}(w \cdot \lambda) \otimes k$ do not coincide with the socle series. However, this seems still to be the case for the filtrations of $H_{f}^{l(w)}(w \cdot \lambda)$ when $\left\langle\alpha^{v}, \lambda\right\rangle<p^{2}-2 p$.

3.3. TYPE $\mathrm{G}_{2}$. In this case we get

$$
\begin{gathered}
H_{t}^{i}(\nu)=0 \text { for all } \nu, \quad \text { for } i \neq 2,3,4,5 \\
\operatorname{ch}\left(H_{t}^{2}(w \cdot \lambda) \otimes k\right)= \begin{cases}\operatorname{ch} H_{k}^{2}(w \cdot \lambda) & \text { if } l(w)=1, \\
\operatorname{ch} H_{k}^{1}(w \cdot \lambda) & \text { if } l(w)=2,3, \\
0 & \text { otherwise. }\end{cases}
\end{gathered}
$$

$$
\operatorname{ch}\left(H_{t}^{3}(w \cdot \lambda) \otimes k\right)= \begin{cases}\operatorname{ch} H_{k}^{3}(w \cdot \lambda)-\operatorname{ch} H_{k}^{4}(w \cdot \lambda) & \text { if } l(w)=2, \\ \operatorname{ch} H_{k}^{2}(w \cdot \lambda)-\operatorname{ch} H_{k}^{1}(w \cdot \lambda) & \text { if } l(w)=3, \\ \operatorname{ch} H_{k}^{2}(w \cdot \lambda) & \text { if } l(w)=4, \\ 0 & \text { otherwise. }\end{cases}
$$


(4)

$$
\operatorname{ch}\left(H_{t}^{4}(w \cdot \lambda) \otimes k\right)= \begin{cases}\operatorname{ch} H_{k}^{4}(w \cdot \lambda) & \text { if } l(w)=2 \\ \operatorname{ch} H_{k}^{4}(w \cdot \lambda)-\operatorname{ch} H_{k}^{5}(w \cdot \lambda) & \text { if } l(w)=3 \\ \operatorname{ch} H_{k}^{3}(w \cdot \lambda)-\operatorname{ch} H_{k}^{2}(w \cdot \lambda) & \text { if } l(w)=4 \\ 0 & \text { otherwise }\end{cases}
$$

$$
\operatorname{ch}\left(H_{t}^{5}(w \cdot \lambda) \otimes k\right)= \begin{cases}\operatorname{ch} H_{k}^{5}(w \cdot \lambda) & \text { if } l(w)=3,4 \\ \operatorname{ch} H_{k}^{4}(w \cdot \lambda) & \text { if } l(w)=5 \\ 0 & \text { otherwise. }\end{cases}
$$

This time one may use Figure 2, p. 256 of [2] to see for which $\nu$ there is nonzero $p$-torsion in $H^{i}(\nu), i=2,3,4,5$. Unfortunately, there are a few inaccuracies in this figure. A corrected version is available from the author upon request.

Contrary to what happened in 3.1 and 3.2 we cannot here derive from our results in $\$ 1$ that the $p$-torsion in $H^{*}(w \cdot \lambda)$ for $\lambda$ satisfying $\left\langle\alpha^{v}, \lambda+\rho\right\rangle\left\langle p^{2}, \alpha \in R\right.$ is killed by $p$ for all $w$. Hence the sum formula is more complicated as we cannot apply Corollary 2.4 but have to apply Theorem 2.3 directly. We leave it to the reader to write down the explicit formulas.

\section{REFERENCES}

1. H. H. Andersen, Cohomology of line bundles on G/B, Ann. Sci. École Norm. Sup. (4) 12 (1979), $85-100$.

2. On the structure of the cohomology of line bundles on G/B, J. Algebra 71 (1981), 245-258.

3. Filtrations of cohomology modules for Chevalley groups, Ann. Sci. École Norm. Sup. (4) 16 (1983), 495-528.

4. K. Kühne-Hausmann, Zur Untermodulstruktur der Weylmoduln für $S L_{3}$, Thesis, Universität Bonn, 1984.

School of Mathematics, Institute for Advanced Study, Princeton, New Jersey 08540

Matematisk Institut, Aarhus Universitet, 8000 Aarhus C, Denmark 